\begin{tabular}{r|l} 
Marc Lescarbot & $\begin{array}{l}\text { MARIE- } \\
\text { CHRISTINE } \\
\text { sur les traces } \\
\text { de Pline l'Ancien }\end{array}$ \\
\end{tabular}

Summary: This study examines the relationship between the work of Pliny the Elder and Marc Lescarbot's Histoire de la Nouvelle-France. Among the many works cited by the erudite lawyer, the annals of the Roman naturalist stand out as constituting a veritable encyclopedia of universal knowledge. Curiously, Lescarbot, who sees in the Natural History a model to imitate, is not afraid to commit to paper the tales and exaggerations that it contains, if only to set himself apart from them. The importance of the French traveller's indebtedness to his Latin predecessor can be explained not only by the authority that the latter enjoyed at the beginning of the Grand Siècle, but also by an agreement of thought: very much like the Roman, the Frenchman from Vervins castigates the deceptive behaviour and the taste for luxury so widespread among his contemporaries.

\title{
Remarques liminaires
}

Marc Lescarbot, auteur d'une Histoire de la Nouvelle-France ${ }^{2}$ plusieurs I fois rééditée, n'a guère besoin de présentations auprès des spécialistes de la Renaissance. Au reste, durant la dernière décennie, sa « chronique américaine » a donné lieu à une floraison d'études critiques que l'on pense aux travaux de Frank Lestringant ${ }^{3}$, de Paolo Carile ${ }^{4}$ et de Guy Poirier ${ }^{5}$ pour n'en citer que quelques-uns. Aussi je m'attarderai peu sur le personnage comme tel qui séjourna en Acadie de 1606 à $1607^{6}$, mais je traiterai plutôt des substrats intertextuels que l'on peut percevoir derrière son vaste plaidoyer en faveur de la colonisation française en Amérique.

Plus d'un chercheur a mis au jour la fréquence des emprunts de Marc Lescarbot. La polémique qui l'opposa aux voyageurs Samuel de Champlain ${ }^{7}$ et Pierre Biard ${ }^{8}$ a déjà fait l'objet d'analyses suffisamment fouillées pour qu'il ne soit besoin d'y revenir en détail. Mon intention n'est pas non plus de 
reprendre ici mes considérations sur l'importance de la digression érudite sous la plume de l'auteur ${ }^{9}$, ni même de donner un aperçu de ses nombreuses lectures comme je l'ai fait récemment ${ }^{10}$. Je me bornerai à examiner le rôle de la référence plinienne dans l'élaboration de l'historiographie acadienne ${ }^{11}$.

Le rapprochement entre Marc Lescarbot et Pline ne va pas de soi, tant s'en faut. Qu'ont en réalité en commun le compagnon de Champlain en Acadie et l'auteur de l'Histoire naturelle, séparés par plus de quinze siècles ? Telle est la problématique que je me propose d'examiner dans le cadre de cette étude.

Que Lescarbot utilise les annales du Romain pour étoffer son enquête ethnographique et son inventaire topographique n'a rien au demeurant de vraiment exceptionnel. Loin d'échapper aux fabulas des Anciens, l'Amérique, terre vierge propice à l'amplification, se trouve depuis les navigations de Colomb au confluent des légendes d'autrefois et de l'expérience des modernes. En parcourant ces régions lézardées de zones d'ombre, voyageurs et chorographes tentent de modeler une matière nouvelle dans un cadre traditionnel, comme l'a montré très justement Frank Lestringant ${ }^{12}$. À l'instar des cosmographes de la Renaissance avec qui il partage une épistémè commune, le compagnon de Poutrincourt érige son Acadie sur des principes hérités de l'Antiquité qu'il évoque dans un jeu complexe d'accréditation ou de réfutation.

Aucun ouvrage, pas même les grandes œuvres de Platon et d'Aristote, n'exerce sur l'avocat de Vervins une influence aussi considérable que l'Histoire naturelle de Pline qu'il cite à profusion comme un texte fondateur du savoir scientifique et de l'ethnologie comparée. Au total, on décompte cinquante renvois explicites à l'ouvrage du naturaliste latin dont trente-neuf allusions dans le sixième livre seulement de la chronique du voyageur. C'est assez dire qu'il s'agit d'une source encyclopédique de prédilection sous la plume de Marc Lescarbot.

\section{Un discours archétypal}

Que Pline, en collationnant ses multiples témoignages, amalgame volontiers des fables à la réalité ne semble nullement entamer son autorité auprès de celui qu'on surnomme parfois « l'Hérodote de la Nouvelle-France » qui lui prête un crédit incontestable jusque dans les domaines les plus hasardeux. Ainsi, les idées de Pline et de certains poètes de son époque sur l'existence des Hespérides reçoivent toute son adhésion. Mieux encore, il n'hésite pas à établir une relation directe entre ces îles mythiques et les Antilles, invoquant l'Histoire naturelle à titre de «preuve » de la justesse de son raisonnement : "volontiers je m'arreteray à ce que le méme Pline, sur une chose pleine d'obscurité, recite ${ }^{13}$ qu'un Statius Sebosus employa quarante jours à 
naviger depuis les Gorgones (qui sont les iles du Cap Verd) jusques aux Hesperides. [... ] Surquoy je conclus que les Hesperides ne sont autres que les iles de Cuba, l'Hespagnole, la Jamaïque, \& autres voisines au golfe de Mexique » $(H N F, \mathrm{I}$, p. 28$)$. On retiendra dans ce passage, faute de pouvoir nous y attarder davantage, le caractère aléatoire de ces spéculations géographiques fondées sur la progression incertaine du navigateur Statius Sebosus et les conjectures de quelques-uns. Soulignons en outre la certitude de notre auteur que suggère l'assertion «surquoy je conclus » et le plaisir engendré par la digression comme l'indique l'expression «volontiers je m'arreteray », propos qui contrastent violemment avec les démentis opposés par le chroniqueur de l'Acadie à l'encontre des affirmations des voyageurs contemporains, évoquées d'ordinaire comme à regret ${ }^{14}$ ou accompagnées de nuances restrictives ${ }^{15}$. Mais l'ascendant de Pline sur Lescarbot ne se limite pas à la géographie : celui-ci lui attribue un discernement en médecine, invoquant comme dignes de foi ses considérations sur les effets néfastes des eaux dormantes :

Quelquefois aussi ce mal [le scorbut] arrive par un vice qui est méme és eaux de fonteines coulantes, comme si elles sont parmi ou prés des marais, ou sortent d'une terre bouëuse, ou d'un lieu qui n'a point l'aspect du Soleil. Ainsi Pline récite qu'au voyage que fit le Prince Cesar Germanicus en Allemagne, [ . . . ] il la fit camper [son armée] [ . . . en un lieu où ne se trouva qu'une seule fontaine d'eau douce, laquelle neantmoins fut si pernicieuse, que tous ceux qui en beurent perdirent les dents en moins de deux ans [ . . . ] $\left(H N F\right.$, IV, p. 468) ${ }^{16}$.

Qui plus est, l'auteur de l'Histoire de la Nouvelle-France prête au compilateur latin une grande perspicacité en ce qui concerne les lois de la physique. Qu'on en juge par ce commentaire sur la formation des tempêtes en mer :

Or ces grains de vents, lesquels autrement on appelle orages, il n'y a danger de dire comme ilz se forment, \& d'où ilz prennent origine. Pline en parle en son Histoire naturele, \& dit en somme que ce sont exhalations \& vapeurs legeres elevées de la terre jusques à la froide region de l'air : \& ne pouvans passer outre [ . . ] $(H N F$, IV, p. 521).

Malgré l'exactitude de la référence, il est clair que Lescarbot infléchit à son gré les assertions de celui que l'on pourrait à juste titre désigner comme son émule. Une simple confrontation de ce passage avec le texte d'origine suffit pour s'en convaincre. Là où l'auteur latin présente ses spéculations comme de pures conjectures : «Je ne nierai pas non plus que des vents, ou plutôt des souffles, ne puissent provenir aussi d'une exhalaison aride et sèche de la terre ${ }^{17}$, Lescarbot y voit une quasi-certitude scientifique comme le suggère la formule introductive : «Ces grains de vents, [ . . ] il n'y a danger de dire 
comme ilz se forment $[\ldots] \gg$. Tout se passe comme si le discours hypothétique de Pline, par le relais de la rhétorique lescarbotienne, se muait en un savoir avéré. Au demeurant, la seule mention de l'ouvrage latin parait ici autoriser la digression : «Pline en parle en son Histoire naturele [ . . . ]», affirme le chroniqueur pour toute justification.

Entre les annales de Lescarbot et celles du Romain se développe une étroite relation de contiguïté. Non seulement les observations du naturaliste autorisent la glose lescarbotienne, mais l'inverse se produit également lors même que le compagnon de Champlain tente d'infirmer par sa propre expérience les dires de son prédécesseur. Ainsi l'extraordinaire longévité des peuples d'Acadie permet de valider celle des habitants de la Taprobane et de Pandore évoquée par Pline ${ }^{18}$ : «Aussi ces peuples vivent-ils un long âge, qui est ordinairement de sept ou huit-vingts ans. [ . . . ] De sorte que ce n'est miracle particulier ce que dit Pline que les Pandoriens vivent deux cens ans, ou que ceux de la Taprobane sont encore alaigres à cent ans » (HNF, VI, p. 859).

Non moins surprenante est la clairvoyance que Lescarbot prête à Pline en ce qui concerne la religion : «Les Payens ont reconu ceci, \& entre autres Pline ${ }^{19}$ quand il a dit que c'est grand signe de divinité à un homme mortel d'ayder \& soulager un autre mortel » (HNF, VI, p. 714). Que penser encore du passage suivant où l'avocat-écrivain paraphrase l'Histoire naturelle à la suite de saint Augustin ${ }^{20}$ pour dénoncer l'iconographie religieuse au sein de l'Église ? : «Et de vérité Pline dit ${ }^{21}$, qu'il n'y a chose qui demontre plus l'imbecillité du sens humain, que de vouloir assigner quelque image ou effigie à Dieu. Car en quelque part que Dieu se montre, il est tout de sens, de veuë, d'oüe, d'ame, d'entendement; et finalement il est tout de soy-méme, sans user d'aucun organe $22 »(H N F$, VI, p. 719). Pareille audace, qui va à l'encontre de toute une tradition ecclésiale, laisse, sur ce point précis, croire à la suprématie de la foi des Païens et des Sauvages, réfractaires à cette imagerie. Faut-il voir dans ce refus de la représentation divine l'influence du protestantisme 23 ?

Quoi qu'il en soit, l'Histoire naturelle permet d'accréditer le témoignage de Lescarbot. Devant le comportement étrange d'un sorcier, l'avocat-écrivain se souvient d'une anecdote semblable racontée par le naturaliste ${ }^{24}$ : «Méme j'ay quelquefois ouï dire que ce maitre diable en ce conflict egratignoit Membertou. Et de ceci me suis souvenu lisant en l'histoire de Pline chose semblable, que ce maître singe égratigne $\&$ bat ses sacrificateurs negligens en leur office » (HNF, VI, p. 728). Cette comparaison met au jour la complémentarité de l'expérience du voyage et de la lecture. En plus d'attester la véracité des affirmations contenues dans le récit anecdotique, le rapprochement interethnique émane ici d'un désir de réhabilitation du Sau- 
vage d'Amérique dont l'étrangeté des coutumes n'a rien à envier à celle des Anciens.

On ne saurait assez insister sur l'influence du traité de Pline dans le récit de Lescarbot. Est-il question de botanique, de zoologie, de physique, de médecine, de morale ou d'ethnographie, l'Histoire naturelle fournit un véritable moule discursif sur lequel viennent se greffer les observations du voyageur. Découvrant une nouvelle plante, Lescarbot emprunte au naturaliste une appellation pour la désigner : " Je croy que ce soient Afrodilles, suivant la description que Pline en fait ${ }^{25} »(H N F$, VI, p. 931). L'Histoire naturelle se dessine, sous sa plume, comme un répertoire encyclopédique dont la chronique acadienne tantôt corrobore les assertions ainsi qu'on l'a vu au sujet des Hespérides notamment ${ }^{26}$, tantôt in contrario s'en démarque ouvertement, ayant alors recours à la technique du repoussoir pour mettre en valeur la simplicité amérindienne, comme en fait foi l'allusion qui suit : « Je laisse aussi les excés qui consistent en meubles, renvoyant le Lecteur à Pline $^{27}$ qui a parlé amplement des pompes \& superfluitez Romanesques [ . . . ]. Je veux seulement parler des Matachiaz de noz Sauvages » $(H N F$, VI, p. 815-16). L'Acadie, dans un constant dialogisme entre les réflexions du naturaliste et les découvertes du Vervinois, devient un territoire exploratoire où sont mises à l'épreuve des spéculations aussi diversifiées que la robustesse des laboureurs ( $H N F$, VI, p. 408), les effets du climat sur l'agilité des peuples $(H N F$, VI, p. 806), la cause d'un certain « Mal de bouche » $(H N F, \mathrm{IV}, \mathrm{p} .468)$, les contes écrits à propos de la rivière Norembègue $(H N F$, IV, p. 486), l'origine des tempêtes maritimes ( $H N F$, IV, p. 521) et le comportement des poissons en hiver (HNF, VI, p. 521). Veut-on comparer les mœurs des Micmacs à celles des hommes d'ailleurs, l'Histoire naturelle devient encore une mention omniprésente; qu'il s'agisse des anciens Italiens $(H N F$, VI, p. 834), des Tartares (HNF, VI, p. 837), des Éthiopiens ( $H N F$, VI, p. 837), des Égyptiens ( $H N F$, VI, p. 843), des Pandoriens (HNF, VI, p. 858) ou des Anglais et des Écossais ( $H N F$, VI, p. 860), la pénétration du Romain ne semble point connaître de limites. Â côté de tous ces renvois explicites, il convient de souligner la présence des réminiscences plus discrètes. Fait-il allusion aux erreurs des géographes à propos du Nil, Lescarbot se garde bien de mentionner Pline dont il s'inspire manifestement : «Ainsi noz Geographes nous font croire que le Nil procede d'un lac qui produit d'autres rivieres, lesquelles se déchargent au grand Ocean » $(H N F$, IV, p. 543 ${ }^{28}$. On pourrait être tenté de justifier cette omission par le refus de porter ombrage à son devancier. Mais comment dès lors expliquer que Lescarbot, si prompt d'ordinaire à afficher ses sources ${ }^{29}$, les taise quand il évoque les semailles de Séranus ( $H N F$, IV, p. 600), anecdote derrière laquelle il est aisé de reconnaître un écho de l'Histoire naturelle ${ }^{30}$ ? Il semble 
en réalité que l'auteur du Théâtre de Neptune soit à tel point imprégné de la chronique du Romain qu'il la paraphrase involontairement.

\section{De timides critiques}

Malgré l'importance de sa dette envers Pline, n'allons cependant pas croire que Lescarbot perde, en disciple aveugle, tout sens critique. Si le Vervinois peut paraître crédule, allant jusqu'à donner foi aux légendes des Anciens, il n'hésite pas cependant à garder ses distances devant certaines affirmations comme celles sur les traits physiques des peuples de la Taprobane ${ }^{31}:$ : Pline donne les mémes qualitez corporeles aux peuples de la Taprobane, disant qu'ils ont les cheveux roux, les ïeux pers, \& la voix horrible \& épouvantable. En quoy je ne sçay si je le doy croire, attendu le climat qui est souz la ligne æquinoctiale » $(H N F, \mathrm{VI}$, p. 804). Au reste, cette marque de dissidence n'est pas unique : Lescarbot s'inscrit encore en faux contre les allégations du naturaliste sur l'origine du toponyme de la Grande Canarie : «noz François découvrirent la grand' Canarie, ainsi appellée (je croy) à cause des Cannes de succre qu'elle produit en abondance, $\&$ non pour-ce qu'elle produit grande quantité de chiens, ainsi que disent Pline et $\operatorname{Solin}^{32} »(H N F$, II, p. 161). Décrivant les peuples de l'Acadie, Lescarbot ne peut résister à la tentation d'écorcher au passage les chimères véhiculées dans les annales pliniennes au sujet de créatures étranges vivant aux confins de l'Inde ${ }^{33}:$ " Au reste il n'y a point parmi eux [les peuples d'Acadie] de ces hommes prodigieux desquels Pline fait mention, qui n'ont point de nez, ou de lévres, ou de langue ; item qui sont sans bouche, n'ayans que deux petits trous, desquels l'un sert pour avoir vent, l'autre sert de bouche : item qui ont des tétes de chiens, \& un chien pour Roy : item qui ont la téte à la poitrine, ou un seul œil au milieu du front, ou un pié plat \& large à couvrir la téte quand il pleut, \& semblables monstres » $\left(H N F\right.$, VI, p. 804-805) ${ }^{34}$. Mais pourquoi évoquer de telles imaginations, si ce n'est pour s'en démarquer et témoigner ainsi de sa bonne foi ? Il est clair que Lescarbot prend d'autant plus plaisir à rapporter toutes ces amplifications qu'elles se situent en porte-à-faux avec son propre discours. L'allusion intertextuelle au même titre que la référence érudite permet d'agrémenter un récit jugé fade. L'historien qui suit les pas incertains de Hannon le Carthaginois et des autres voyageurs légendaires semble presque déplorer en comparaison la banalité de son expérience américaine et les recherches infructueuses du sieur De Monts en quête d'un meilleur site pour la colonie : « [il] n'y a rien de remarquable (du moins qu'on ait veu au dehors des terres) qu'une riviere de laquelle plusieurs ont écrit des fables à la suite l'un de l'autre, de mémes que ceux qui sur la foy des Commentaires de Hanno, Capitaine Carthaginois, avoient feint des villes en grand nombre par lui baties sur la cóte de l'Afrique qui est arrousée de l'Ocean ${ }^{35} »(\mathrm{HNF}, \mathrm{IV}, \mathrm{p}$. 
486). Même récusée, la digression savante permet en quelque sorte de relever une relation de séjour dépourvue de péripéties et un inventaire sans grande surprise. En évoquant les légendes dont Pline se fait le relais, le chroniqueur renoue, au moyen de la dénégation, avec le mythe, auquel l'écriture du voyage est sans cesse confrontée. La référence plinienne participe ainsi d'une troublante dialectique de la vérité et du mensonge, dans laquelle le discours viatique - pour reprendre l'expression désormais consacrée à ce «genre » littéraire — se trouve par définition engagé.

\section{Une filiation idéologique}

Mais comme les sources susceptibles de piquer la curiosité des lecteurs ne manquent pas, à quoi faut-il dès lors attribuer cette prédilection de Lescarbot pour Pline ? On pourrait d'emblée évoquer l'extraordinaire popularité de l'auteur à l'orée du Grand Siècle. À cet égard, on ne peut que souscrire au jugement de Robert Lenoble sur la « fortune inouïe » de Pline ${ }^{36}$. Mais il y a plus. Le Romain et Lescarbot semblent pétris de la même pâte : leur soif de connaissances les amène à embrasser tous les sujets. L'un comme l'autre n'ont rien d'explorateurs méthodiques, passant d'évidence plus de temps dans leur bibliothèque que dans les contrées lointaines qu'ils décrivent très souvent par procuration. Puisque, de son propre aveu, l'avocat vervinois ne s'aventura guère au-delà des limites de Sainte-Croix ${ }^{37}$, suppléant à cette fixité par une vaste documentation, il fait figure, à l'instar de Pline, de compilateur. À coup de références érudites, le discours viatique s'érige tout autant en un métadiscours qu'en un compte rendu fidèle de voyage. Dresser l'inventaire bibliographique de Lescarbot tiendrait de l'exploit tant les substrats textuels de son œuvre sont multiples, allant de la littérature gréco-romaine aux poèmes de Ronsard, en passant par les histoires et les chansons populaires ${ }^{38}$.

Plus qu'un fonds inépuisable d'anecdotes, Lescarbot voit dans l'Histoire naturelle un modèle à imiter : "Pline \& autres geographes n'estiment point étre hors de leur sujet d'écrire de cette façon » (HNF, III, p. 214), affirme-t-il pour légitimer la verbosité de son discours. Cette émulation ouverte repose aussi sur des affinités qui dépassent le cadre scriptural. Je ne pense pas me tromper en présentant Lescarbot comme un disciple de Pline. En effet, on observe chez l'un et l'autre un culte immuable du naturel. À la suite du Romain, l'annaliste de la Nouvelle-France vitupère tous les excès, qu'il s'agisse de la fatuité des femmes qui se couvrent de bijoux ou du goût du luxe de certains peuples: "L'avarice des hommes a fait qu'on ne trouve point un païs bon s'il n'y a des Mines d'or» $(H N F, \text { VI, p. 937 })^{39}$. Au même titre que Pline, Lescarbot fustige ouvertement la dépravation des voyageurs qui ne prennent plus la mer pour le seul désir de connaître mais qui sont au contraire animés par l'appât du gain : « les delices ont appoltronni \& l'un \& 
l'autre sexe » $(H N F$, I, p. 22), conclut-il plein de dépit devant le peu d'empressement des Français à prendre le large. En outre, comment ne pas reconnaître dans les critiques de Lescarbot à l'endroit de l'avidité des Espagnols et des Portugais ${ }^{40}$ l'anathème lancé quinze siècles plus tôt par Pline : « Ce sont les mœurs qui ont déchu, et non les récompenses. La mer est ouverte dans toute son étendue, tous les rivages sont hospitaliers ; mais la foule immense qui navigue le fait pour l'amour du gain et non de la science ${ }^{41}$. Sans rejeter les bienfaits de la civilisation, Lescarbot comme son prédécesseur condamne le superflu. Reconnaissant chez les Micmacs la félicité du premier âge, il applaudit devant l'absence de tribunaux et d'avocats sur leur territoire : "Il n'y a ny procés, ni auditoires entre eux, ainsi que Pline dit des insulaires de la Taprobane, en quoy il les repute particulierement heureux de n'étre tourmentez de cette gratelle » $\left(H N F\right.$, VI, p. 888) ${ }^{42}$. Au fil de cette rhétorique menant à l'idéalisation du primitif, tout ce qui s'écarte de la voie du naturel se voit rejeté d'emblée. Pline, en se faisant, à l'instar d'Hésiode, l'apôtre de la simplicité, d'un rêve rustique, en même temps qu'il se présente comme un être tourmenté en quête perpétuelle de découvertes, ne pouvait faire autrement que de baliser la voie du premier anthropologue de la Nouvelle-France. Son invocation finale, au terme du livre XXXVII de son histoire : «Salut, Ô Nature, mère de toutes choses ! et daigne m'être favorable, à moi qui, seul entre tous [ . . . ], t'ai complètement célébrée ! » pourrait bien figurer en exergue à l'ouvrage de Marc Lescarbot, pour qui l'ordre naturel semble incarner les ferments de toute réflexion éthique.

Au-delà de cet étroit cousinage entre les deux hommes, au-delà de ce rejet commun de l'artifice, la référence intertextuelle permet en quelque sorte de recréer chez Lescarbot une Acadie métissée où cohabitent les traits de l'Antiquité et d'une civilisation nouvelle. En définitive, par ce constant entrelacement des textes s'effectue un voyage aller et retour entre le maintenant et le jadis, entre l'Amérique et les vieux continents qui permet de transfigurer, voire de légendariser l'Amérique sauvage, terre riche de promesses. Par la multiplication des références encyclopédiques, Lescarbot n'espère rien de moins que de « ramener le siecle d'or » en Nouvelle-France (HNF, VI, p. 933) ${ }^{43}$. Évoquant son prédécesseur comme repère axiologique, il avouera même dans son «A-Dieu à la Nouvelle France » préférer sa bienheureuse Acadie aux « beautez [ . . . ] dignes des bien-heureux $»^{44}$ du pays légendaire de la Taprobane, comme quoi les réminiscences livresques qui affleurent derrière la plupart des écrits lescarbotiens contribuent à la transposition en sol amérindien d'une Arcadie $^{45}$ mythique, peuplée non plus de bergers mais de vigoureux Armouchiquois, exempts de tous les maux de la vieille Europe ${ }^{46}$.

Université York 


\section{Notes}

1. Pour mener mes recherches sur les écrits de Marc Lescarbot, j'ai bénéficié du soutien financier du «SSHRC Travel and Small Grants Sub-Committee » de la Faculté des lettres de l'Université York.

2. Toutes les références de Marc Lescarbot dans cette étude renvoient à l'Histoire de la Nouvelle-France, Paris, Adrien Périer, 1618, en abrégé $H N F$ dans le texte.

3. Frank Lestringant, "Champlain, Lescarbot et la "Conférence" des histoires » dans Scritti sulla Nouvelle-France nel seicento, Quaderno del Seicento francesi, $n^{\circ}$ 6, Bari, Adriatica, 1984, p. 69-88.

4. Paolo Carile, Le Regard entravé. Littérature et anthropologie dans les premiers textes sur la Nouvelle-France, Sillery (Québec), Septentrion/Rome, A. Editrice, 2000. À la fin de son ouvrage, l'auteur dresse un bilan de « la fortune critique de Lescarbot » (p. 213-18).

5. Guy Poirier, « Marc Lescarbot au pays des Ithyphalles », Renaissance and Reformation/Renaissance et Réforme, ns XVII, 3, 1993, p. 73-85.

6. Sur la vie de Marc Lescarbot, l'on reportera le lecteur à l'ouvrage d'Éric Thierry, Marc Lescarbot (vers 1570-1641). Un homme de plume au service de la Nouvelle-France, Paris, H. Champion, 2001.

7. Voir sur cette question l'analyse de Frank Lestringant, « Champlain, Lescarbot et la "Conférence" des histoires », p. 69-88.

8. Les différends opposant Lescarbot au jésuite Pierre Biard ont fait l'objet d'un chapitre de Paolo Carile ( «Lescarbot et Biard : la première querelle de l'évangélisation », $o p$. cit., p. 129-49).

9. On lira sur cette question notre article intitulé «Les méandres de Marc Lescarbot au Nouveau Monde : du voir-dire à la digression érudite », dans Miroirs de textes. Récits de voyage et intertextualité, Nice, Publications de la Faculté des Lettres, Arts et Sciences Humaines de Nice, ns no 49, 1998, p. 187-99.

10. «Le gai savoir ou la quête épistémologique de Marc Lescarbot au Nouveau Monde », étude à paraître dans un numéro spécial de La Revue française sous la direction de Dominique Lanni, consacré à la culture du voyageur à l'âge classique.

11. Bien que j'aie déjà suggéré l'importance des emprunts de Lescarbot au naturaliste latin dans les deux articles précités aux notes 9 et 10, ce parallélisme n'a jamais fait l'objet, à ma connaissance, d'une étude suivie.

12. Frank Lestringant, L'Atelier du cosmographe ou l'image du monde à la Renaissance, Paris, A. Michel, 1991, p. 70-73.

13. Pline, VI, 36. Les citations de Pline l'Ancien sont extraites de l'Histoire naturelle de Pline, trad. M. É. Littré, 2 vol., Paris, Dubochet, Le Chevalier, 1851.

14. À titre d'exemple, rapportons les formules prétéritives du chroniqueur pour introduire ses considérations sur la ville de Norumbega : "Sans donc amener ce qu'ont dit les premiers Hespagnols \& Portugais » $(H N F$, IV, p. 486) et les présumés miracles rapportés par le jésuite Pierre Biard : « Je serois trop long si je voulois particulariser tout ce qui se pourroit rapporter en ce sujet » $(H N F, \mathrm{~V}$, p. 661).

15. Ainsi le jugement du découvreur du Canada sur le maquillage des veuves se voit assorti de certaines limites : «je ne veux affermer que ce qu'en a écrit Jacques Quartier soit general » $(H N F, \mathrm{VI}, \mathrm{p} .832)$.

16. Pline, $\mathrm{XXV}, 6$. 
17. Pline, II, 44.

18. Pline, VII, 2.

19. Pline, II, 5.

20. Saint Augustin écrit : «Il [Varron] ajoute que, pendant plus de cent soixante-dix ans, les anciens Romains adorèrent les Dieux sans user de représentations » (La Cité de Dieu, trad. G. Combès, dans CEuvres de saint Augustin, Paris, Desclée de Brouwer, 1959, t. 33, livre IV, chap. 31, p. 631).

21. Pline, II, 5.

22. En italique dans le texte.

23. La question de la spiritualité du voyageur a notamment été abordée par P. Beuzart dans «La Religion de Marc Lescarbot de Vervins, explorateur au Canada », Bulletin de la Société de l'histoire du protestantisme français, vol. LXXXVII, 1938, p. 237-60.

24. Pline, II, 5.

25. Pline, XXI, 68.

26. Lorsqu'il décrit les cétacés qu'il n'a probablement pas eu le loisir d'observer en Acadie, Lescarbot cite à nouveau l'Histoire naturelle : «Pline dit qu'és Indes il s'en trouve [des baleines] qui ont quatre arpens de terre de longueur » $(H N F$, VI, p. 917). Voir Pline, IX, 2.

27. Pline, XXXIII, 51.

28. Pline écrit : «La source (autant qu'ont pu s'étendre les recherches du roi Juba) en est dans une montagne de la Mauritanie inférieure, non loin de l'Océan ; il forme aussitôt un lac qu'on appelle Nilis » $(\mathrm{V}, 10)$.

29. Il importe de préciser que d'ordinaire l'annaliste mentionne dans la marge non seulement le titre mais le chapitre des ouvrages auxquels il fait référence.

30. Pline, XVIII, 5.

31. Pline, VI, 24.

32. Pline, VI, 36. Cf. Caïus Julius Solin, Polyhistor, trad. M. A. Agnant, Paris, Panckoucke, 1847 , chap. 57, p. 353.

33. Pline, VI, 35 ; VII, 2.

34. Loin d'être originale, cette attaque contre les erreurs de Pline concernant l'existence d'êtres monstrueux fait partie, au dire de Frank Lestringant, de la « doxa géographique du XVI ${ }^{\mathrm{e}}$ siècle ». Jean de Léry, notamment, s'en est pris aux cyclopes et autres créatures difformes mentionnés dans la somme du naturaliste (Histoire d'un voyage faict en la terre du Brésil, éd. Frank Lestringant, coll. «Bibliothèque classique », Paris, Le Livre de Poche, 1994, p. 220-21, note 3).

35. Pline, V, 1.

36. Robert Lenoble, Esquisse d'une histoire de l'idée de Nature, coll. «Évolution de l'humanité », Paris, A. Michel, 1969, p. 137.

37. $C f$. : « Je ne sçay à quel propos Champlein en la relation de ses voyages imprimée l'an mille six cens treize, s'amuse à écrire que je n'ay point été plus loin que Sainte-Croix, veu que je ne di pas le contraire » (HNF, IV, p. 594).

38. On retrouve notamment une strophe du Châtelain de Coucy, un chansonnier du Moyen Âge (HNF, VI, p. 803). 
39. Sur les intérêts mercantiles des commerçants, qui favorisent les expéditions d'outremer, Lescarbot ne ménage pas ses mots : « on voudroit trouver les thresors d'Atabalippa [Atahualpa, le dernier empereur inca] sans travail \& sans peine, mais on y vient trop tard, \& pour en trouver il faut chercher, il faut faire de la dépense, ce que les grans ne veulent pas. Les demandes ordinaires que l'on nous fait, sont : Y a-[t-]il des mines d'or \& d'argent ? et personne ne demande, Ce peuple là est-il disposé à entendre la doctrine Chrétienne? » $(H N F, \mathrm{I}, \mathrm{p} .17)$.

40. $C f$. : «La méme convoitise a eté l'aiguillon qui depuis six-vints ans a poussé les Portugais, Hespagnols, \& autres peuples de l'Europe à se hazarder sur l'Ocean, chercher des nouveaux mondes deça \& dela l'Equateur, \& en un mot environner la terre ; laquelle aujourd'huy se trouve toute reconuë par l'obstinée \& infatigable avidité de l'homme, excepté quelques cotes antarctiques, \& quelques-unes à l'Occident outre l'Amerique, léquelles ont été negligées, parce qu'il n'y avoit rien à butiner » $(H N F, \mathrm{I}$, p. 2-3).

41. Pline, II, 45.

42. Pline, VI, 24.

43. Plus loin, Lescarbot avoue son désir de renouer avec d'anciennes coutumes romaines : « il faut renouveller les antiques Corones d'epics de blé, \& faire que la premiere gloire soit celle que les anciens Romains appelloient Gloria adorea, gloire de froment, afin d'inviter chacun à bien cultiver son champ, puis que la terre se presente liberalement à ceux qui n'en ont point » (HNF, VI, p. 933).

44. Les Muses de la Nouvelle-France, Paris, A. Périer, 1618, p. 31.

45. Même si le thème n'apparaît pas dans l'Histoire de la Nouvelle-France, le toponyme Arcadie est encore utilisé par Samuel de Champlain dans Des Sauvages (éd. A. Beaulieu et R. Ouellet, Montréal, Typo, 1993, p. 81).

46. Sur cette vision idéalisée du pays et de ses habitants, on renverra le lecteur à l'étude d'Éric Thierry intitulée «Marc Lescarbot et la vision édénique de l'Acadie », Études canadiennes, $\mathrm{n}^{\mathrm{O}} 37,1994$, p. 339-53. 